\title{
Altered Expression of the m6A Methyltransferase METTL3 in Alzheimer's Disease
}

\author{
He Huang, ${ }^{1}$ Judith Camats-Perna, ${ }^{1}$ Rodrigo Medeiros, ${ }^{1,2}$ ๑Victor Anggono, ${ }^{1}$ and ${ }^{D J o c e l y n ~ W i d a g d o}{ }^{1}$
}

\author{
https://doi.org/10.1523/ENEURO.0125-20.2020
}

${ }^{1}$ Clem Jones Centre for Ageing Dementia Research, Queensland Brain Institute, The University of Queensland, Brisbane, Queensland 4072, Australia and ${ }^{2}$ Institute for Memory Impairments and Neurological Disorders, University of California, Irvine, Irvine, CA 92697

\begin{abstract}
Cognitive impairment in Alzheimer's disease $(A D)$ is associated with dysregulation of the RNA and protein expression profiles in the brain. Recent studies have highlighted the importance of RNA post-transcriptional regulation (epitranscriptomics) in higher order brain functions. Specifically, N6-methyladenosine (m6A), which controls RNA stability, splicing, translation and trafficking, plays an important role in learning and memory. This raises the question of whether m6A signaling is perturbed in AD. To address this, we investigated the expression profile of known m6A-regulatory genes using a public RNA-seq dataset and identified a subset of genes which were significantly dysregulated in the human $A D$ brain. Among these, genes encoding the m6A methyltransferase, METTL3, and a member of the m6A methyltransferase complex (MACOM), RBM15B, were downregulated and upregulated in the hippocampus, respectively. These findings were validated at the protein level using an independent cohort of postmortem human brain samples. Unexpectedly, we observed an accumulation of methyltransferase-like 3 (METTL3), but not RBM15B, in the insoluble fractions, which positively correlated with the levels of insoluble Tau protein in the postmortem human AD samples. Aberrant expression and distribution of METTL3 in the hippocampus of the AD brain may therefore represent an epitranscriptomic mechanism underlying the altered gene expression patterns associated with disease pathogenesis.
\end{abstract}

Key words: Alzheimer's disease; epitranscriptomic; METTL3; N6-methyladenosine; neurodegenerative disorders; RNA methylation

\section{Significance Statement}

N6-methyladenosine (m6A), the most prevalent internal RNA modification in eukaryotes, controls various aspects of RNA metabolism. m6A plays an important role in learning and memory; however, whether m6A signaling is perturbed in Alzheimer's disease (AD) remains unknown. Here we report a decrease in the messenger RNA (mRNA) expression that encodes the key m6A methyltransferase enzyme, METTL3, in the postmortem hippocampal tissues of AD patients. We also identified a striking alteration in the methyltransferase-like 3 (METTL3) protein expression, including enhanced insolubility and immunoreactivity in the AD hippocampus. This suggests that perturbation of $\mathrm{m6A}$ signaling may present a novel cellular mechanism underpinning dysregulation of gene expression associated with AD pathophysiology.

\section{Introduction}

Alzheimer's disease (AD) is a chronic neurodegenerative disorder that is characterized by a progressive decline in higher cognitive function and memory loss (Tarawneh

\footnotetext{
Received April 1, 2020; accepted August 17, 2020; First published August 26, 2020.

The authors declare no competing financial interests.
}

and Holtzman, 2012). Accumulation of insoluble neurotoxic aggregates, including amyloid- $\beta(\mathrm{A} \beta)$ plaques and intracellular Tau neurofibrillary tangles, represents a major pathologic hallmark of $A D$ that leads to synaptic dysfunction and

Author contributions: J.W. conceived the project; H.H., J.C-P., R.M., V.A., and J.W. designed research and analyzed data; H.H. performed research; H.H., V.A., and J.W. wrote the paper. All authors discussed the results and commented on the manuscript. 
ultimately neuronal death and dementia (Polanco et al., 2018). Changes in gene and protein expression profiles underpin dysfunction in many fundamental cellular processes during disease pathogenesis in AD (Neueder, 2019). One of the mechanisms underlying $A D$-related changes in gene expression involves perturbations of the epigenome via disease-specific changes in chromatin structure and/or transcription programs (Sanchez-Mut and Graff, 2015). These include alterations in DNA methylation (De Jager et al., 2014; Lunnon et al., 2014) and histone modifications (Marzi et al., 2018; Nativio et al., 2018; Klein et al., 2019).

In recent years, chemical modifications in RNA have emerged as important mechanisms for the control of gene expression and protein translation (Saletore et al., 2012; Zhao et al., 2017). N6-methyladenosine (m6A), the most abundant and reversible post-transcriptional modification on eukaryotic messenger RNAs (mRNAs), is a versatile regulator of mRNA stability, splicing, localization, and translation rate (Schwartz, 2016; Zhao et al., 2017). In mammals, covalent attachment of a methyl group to an adenosine at the $N 6$ position is catalyzed by the multiprotein m6A methyltransferase complex (MACOM) that comprises the catalytic subunit methyltransferase-like 3 (METTL3) which forms a heterodimer with METTL14 (Liu et al., 2014). The precise localization of the METTL3/14 complex is determined by the Wilm's tumor 1 -associating protein (WTAP; Ping et al., 2014). Additional co-factors in the MACOM which facilitate its anchoring and targeting in nuclear speckles and $U$-rich regions adjacent to $\mathrm{m} 6 \mathrm{~A}$ sites in mRNAs include Vir-like m6A methyltransferase associated (VIRMA), zinc finger CCCH-type containing 13 (ZC3H13), Casitas B-lineage lymphoma-transforming sequence-like protein 1 (CBLL1; also known as HAKAI), RNA binding motif protein 15 (RBM15), and RBM15B (Huang et al., 2020b). Recognition of m6A by m6A readers, specific RNA binding proteins that reside in either the nucleus or the cytoplasm dictates the processing outcome of the methylated transcripts (Yang et al., 2018; Shi et

This work was supported by the Australian Research Council (ARC) Discovery Early Career Research Award DE170100112, the University of Queensland (UQ) Early Career Researcher Grant 1834545, and a UQ Amplify Fellowship (J.W.) as well as Australian National Health and Medical Research Council Fellowship and Project Grants GNT112919, GNT1128436, and GNT1139469 (to R.M.) and funding from the Clem Jones Centre for Ageing Dementia Research (R.M. and V.A.). H.H. is a recipient of a UQ Research Training Scholarship. Imaging was performed at the Queensland Brain Institute's Advanced Microscopy Facility using a bright-field slide scanner microscope supported by the ARC Linkage, Infrastructure, Equipment and Facilities Grant LE100100074.

Acknowledgements: We thank Dr. Tee Jong Huat and Tessa Onraet for their assistance in the preparation of human brain extracts and immunohistochemistry as well as Rowan Tweedale for editing this manuscript. The authors also thank the National Institutes of Health/National Institute on Aging-funded Alzheimer's Disease Research Center of the University of California, Irvine (Grant P50 AG16573) and Prof. Jürgen Götz (The University of Queensland, Australia) for providing the human brain samples and Tau-V5 constructs, respectively.

Correspondence should be addressed to Jocelyn Widagdo at j.widagdo@ uq.edu.au.

https://doi.org/10.1523/ENEURO.0125-20.2020

Copyright (C) 2020 Huang et al.

This is an open-access article distributed under the terms of the Creative Commons Attribution 4.0 International license, which permits unrestricted use, distribution and reproduction in any medium provided that the original work is properly attributed. al., 2019). Removal of the methyl groups is mediated by two distinct $\mathrm{m} 6 \mathrm{~A}$ demethylating enzymes, namely the fat mass and obesity-associated (FTO) protein and AlkB homolog 5 (ALKBH5; Jia et al., 2011; Zheng et al., 2013).

The widespread presence of $\mathrm{m} 6 \mathrm{~A}$ in the neuronal transcriptome underpins its numerous functional roles throughout development and adult brain function (Widagdo and Anggono, 2018; Flamand and Meyer, 2019; Livneh et al., 2020). Accumulating evidence has demonstrated essential roles of $\mathrm{m} 6 \mathrm{~A}$ signaling in learning and memory by facilitating the translation of plasticity-related genes in the mouse brain (Widagdo et al., 2016; Li et al., 2017; Walters et al., 2017; Koranda et al., 2018; Shi et al., 2018; Zhang et al., 2018). Given that learning and memory impairments are clinical hallmarks of $A D$, we posited that dysregulation of $m 6 A$ signaling may be associated with the pathophysiology of $A D$. By examining the expression profiles of $\mathrm{m} 6 \mathrm{~A}$ regulatory genes in postmortem human AD brains, we report the aberrant expression of MACOM proteins, including METTL3 and RBM15B, in the hippocampus, indicating a potential perturbation in $\mathrm{m} 6 \mathrm{~A}$ signaling that may contribute to neuronal dysfunction in $A D$.

\section{Materials and Methods}

\section{DNA constructs and antibodies}

The plasmid encoding Mettl3 was generated by amplifying total mouse brain CDNA using the forward primer $5^{\prime}$ ATAGTCGACGATGTCGGACACGTGGAGCTCT-3' and the reverse primer 5'- TTGCGGCCGCGTGCGTCTATAAATT CTTAGGTT- ${ }^{\prime}$. The PCR products were digested with Sall and Notl (New England Biolabs) and ligated into the cut pRK5-myc vector. Plasmids encoding V5-tagged Tau (wildtype and P301L mutant) were generously provided by Prof. Jürgen Götz (Li and Götz, 2017).

Antibodies were purchased from commercial sources as follows: anti-METTL3 (ab195352, Abcam), anti-RBM15B (2249-1-AP, Proteintech), anti-METTL14 (HPA038002, Sigma), anti-Tau (MN1000, Thermo Scientific), anti-V5 (V8137, Sigma or clone7/4, Biolegend), anti-myc (clone 9E10, Bio-Rad Laboratories or clone A-14, Santa Cruz Biotechnology), anti-MAP2 (ab92434, Abcam), or anti$\beta$-actin (sc-47 778, Santa Cruz Biotechnology).

\section{In silico transcriptomic database analyses}

To investigate the expression of m6A-related genes in the postmortem AD brain of male and female subjects, we analyzed a publicly available transcriptomic dataset from the Aging, Dementia and TBI Study (http://aging.brainmap.org) by the Allen Institute for Brain Science (Miller et al., 2017). The demographic and clinical information of donors associated with this study is summarized in Table 1. Adjusted normalized fragments per kilobase of transcript per million (FPKM) mapped reads were used to assess differential gene expression.

\section{Human postmortem brain tissue}

Postmortem brain tissues from cognitively normal and $A D$ individuals (males and females) were obtained from the neuropathology core of the Alzheimer's Disease Research 
Table 1: Demographic summary of donors associated with the Allen Institute's Aging, Dementia and TBI Study

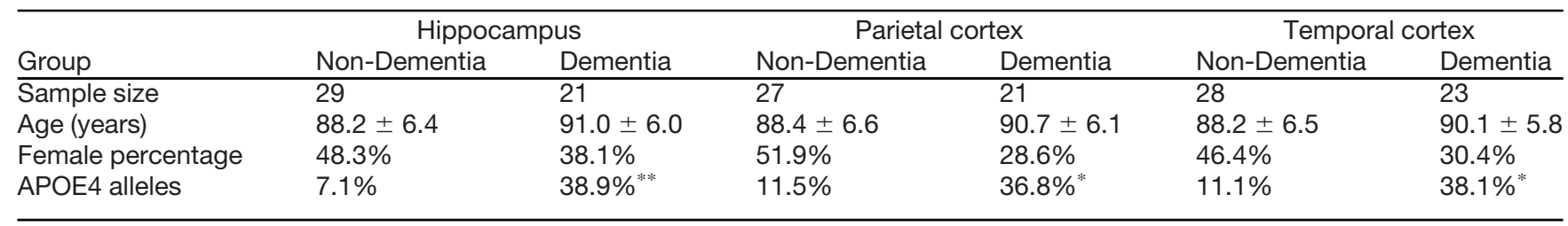

$* p<0.05$

$* * p<0.01, \chi^{2}$ test for gender and APOE4 composition among groups. An unpaired $t$ test was used for age analysis.

Center at the University of California, Irvine (for subject details, see Table 2 and Extended Data Table 2-1). The protocols for obtaining these tissues complied with all guidelines with special respect for donor identity confidentiality and informed consent and were approved by the University of California, Irvine Institutional Review Board. Paraformaldehyde-fixed and frozen hippocampal tissues were provided for this study. Experiments were performed according to the approval granted by the University of Queensland Human Research Ethics Committee (\#2017000490).

\section{Protein extraction and Western blot analysis}

Frozen hippocampal tissues were ground into powder in liquid nitrogen, transferred into a Precellys homogenization tube (Bertin Technologies) and lysed in ice-cold TPER extraction buffer (Thermo Scientific) containing protease and phosphatase inhibitors as described previously (Zumkehr et al., 2015). Briefly, tissue lysates were cleared by ultracentrifugation at $100,000 \times g$ for $60 \mathrm{~min}$ at $4^{\circ} \mathrm{C}$ to obtain the soluble fractions. The resulting pellet was resuspended with $70 \%$ formic acid and again homogenized, followed by another ultracentrifugation step at $100,000 \times g$ for $60 \mathrm{~min}$ at $4^{\circ} \mathrm{C}$. The supernatant was collected as the insoluble fraction. The protein concentration was determined using the Bradford assay (Bio-Rad Laboratories). After normalizing the protein concentration, samples were mixed with $3 \times$ SDS sample buffer and denatured at $95^{\circ} \mathrm{C}$ for $10 \mathrm{~min}$. Equal amounts of protein were loaded on $10 \%$ acrylamide gels and transferred to polyvinylidene fluoride membranes. The membranes were then blocked, washed and incubated with the specific primary antibodies overnight. After washing and incubation with horseradish peroxidase-conjugated secondary antibodies, detection with enhanced chemiluminescence was performed using an Odyssey Fc imaging system (LiCOR). Image Studio software (Li-COR) was used to acquire images and perform densitometry analyses. Quantification of the $A \beta$ levels in the T-PER insoluble

Table 2: Demographic summary of donors from the Alzheimer's Disease Research left at University of California, Irvine

\begin{tabular}{llll}
\hline Group & Control & AD & $p$ value \\
\hline Sample size & 19 & 26 & - \\
Age (years) & $90.3 \pm 4.4$ & $88.6 \pm 6.1$ & 0.31 \\
Female percentage & $52.6 \%$ & $53.8 \%$ & 0.94 \\
APOE4 alleles & $31.6 \%$ & $46.2 \%$ & 0.32
\end{tabular}

$\overline{\chi^{2} \text { test for gender and APOE4 composition among groups. An unpaired } t \text { test }}$ was used for age analysis. fractions was performed using ELISA as described previously (Medeiros et al., 2014).

\section{Co-immunoprecipitation assays}

Co-immunoprecipitation assays were conducted according a previously published protocol (Anggono et al., 2013). Briefly, HEK293T cells were cultured in DMEM containing $10 \%$ fetal bovine serum, $50 \mathrm{U} / \mathrm{ml}$ penicillin, and $50 \mu \mathrm{g} / \mathrm{ml}$ streptomycin at $37^{\circ} \mathrm{C}$ in a humidified $5 \% \mathrm{CO}_{2}$ tissue culture incubator. Cells were transfected using the calcium phosphate precipitation method and lysed $48 \mathrm{~h}$ later with ice-cold cell lysis buffer (1\% Triton X-100, $1 \mathrm{~mm}$ EDTA, 1 mm EGTA, 50 mm NaF, and 5 mm Na-pyrophosphate in PBS) containing protease inhibitors. Cell lysates were cleared at $17,000 \times g$ for $20 \mathrm{~min}$ at $4^{\circ} \mathrm{C}$ and incubated with antibodies coupled to protein A-Sepharose overnight at $4^{\circ} \mathrm{C}$. Beads were washed extensively with ice-cold cell lysis buffer and eluted with $2 \times$ SDS sample buffer. Bound proteins were resolved by SDS-PAGE followed by Western blot analysis.

\section{Immunohistochemistry}

Paraformaldehyde-fixed hippocampal tissues were sliced into $20-\mu \mathrm{m}$ sections using a sliding microtome (SM1020R, Leica). The immunohistochemistry procedure was performed as previously described (Medeiros et al., 2014), including an antigen retrieval step (Tris/EDTA pH 9.0 at $97^{\circ} \mathrm{C}$ for $\left.10 \mathrm{~min}\right)$. Staining with anti-METTL3 antibody (ab195352, Abcam, 1:50 dilution) was performed overnight at $4^{\circ} \mathrm{C}$, followed by detection with biotinylated horse anti-rabbit IgG (Vector Laboratories), avidin-biotin complex (Vector Laboratories), and diaminobenzidine (DAB) staining. Stained hippocampal sections were imaged with a Zeiss Axio Imager Z2 microscope equipped with a $20 \times$ objective and a Metafer slide scanning platform. Quantification of the optical density (OD) of images was performed with Fiji software (ImageJ, NIH). Briefly, color deconvolution of the images was achieved using the set $O D$ vectors for $D A B$ and hematoxylin ( $H D A B)$. The OD value of the image presenting $D A B$ was calculated using the formula $O D=\log (\max$ intensity/mean intensity). Selected regions from CA1, CA2, CA3, and the dentate gyrus (DG) were measured separately for each section.

\section{Immunofluorescence staining}

Sections were first blocked with 5\% normal serum, 2\% BSA, and $0.1 \%$ Tween 20 in TBS at room temperature. Primary antibody incubation was performed overnight at 
Table 3: Statistical summary of the mRNA and protein changes associated with AD brain tissues

\begin{tabular}{|c|c|c|c|c|c|}
\hline Figure & Data & Data structure (normality test) & Type of test & Power & $p$ value \\
\hline \multirow[t]{3}{*}{$\overline{1 A}$} & METTL3 (HIP) & Yes & Permutation $t$ test & {$[95 \% \mathrm{Cl}-0.75,-0.03]$} & 0.049 \\
\hline & METTL3 (PCX) & Yes & Permutation $t$ test & {$[95 \% \mathrm{Cl}-0.63,0.12]$} & 0.204 \\
\hline & METTL3 (TCX) & Yes & Permutation $t$ test & {$[95 \% \mathrm{Cl}-0.63,0.15]$} & 0.220 \\
\hline \multirow[t]{3}{*}{$1 B$} & RBM15B (HIP) & Yes & Permutation $t$ test & {$[95 \% \mathrm{Cl} 0.06,0.83]$} & 0.040 \\
\hline & RBM15B (PCX) & Yes & Permutation $t$ test & {$[95 \% \mathrm{Cl} 0.09,0.81]$} & 0.032 \\
\hline & RBM15B (TCX) & Yes & Permutation $t$ test & {$[95 \% \mathrm{Cl} 0.14,0.83]$} & 0.012 \\
\hline $2 B$ & Soluble METTL3 & No & Permutation $t$ test & {$[95 \% \mathrm{Cl}-0.75,-0.05]$} & 0.030 \\
\hline $2 C$ & Soluble RBM15B & No & Permutation $t$ test & {$[95 \% \mathrm{Cl} 0.11,0.85]$} & 0.036 \\
\hline $2 D$ & Soluble METTL14 & Yes & Permutation $t$ test & {$[95 \% \mathrm{Cl}-0.74,0.02]$} & 0.074 \\
\hline $2 E$ & Soluble tau & No & Permutation $t$ test & {$[95 \% \mathrm{Cl}-0.33,0.08]$} & 0.219 \\
\hline $3 B$ & Insoluble METTL3 & No & Permutation $t$ test & {$[95 \% \mathrm{Cl} 1.27,5.31]$} & 0.012 \\
\hline $3 C$ & Insoluble tau & No & Permutation $t$ test & {$[95 \% \mathrm{Cl} 5.53,18.76]$} & 0.003 \\
\hline \multirow[t]{4}{*}{$4 D$} & $\mathrm{IHC}(\mathrm{CA} 1)$ & Yes & Permutation $t$ test & {$[95 \% \mathrm{Cl} 0.48,5.15]$} & 0.015 \\
\hline & $\mathrm{IHC}$ (CA2) & No & Permutation $t$ test & {$[95 \% \mathrm{Cl} 0.42,1.87]$} & 0.014 \\
\hline & $\mathrm{IHC}(\mathrm{CA} 3)$ & Yes & Permutation $t$ test & {$[95 \% \mathrm{Cl} 0.25,2.11]$} & 0.025 \\
\hline & $\mathrm{IHC}(\mathrm{DG})$ & Yes & Permutation $t$ test & {$[95 \% \mathrm{Cl} 0.36,1.70]$} & 0.007 \\
\hline
\end{tabular}

$\mathrm{HIP}=$ hippocampus; $\mathrm{PCX}=$ parietal cortex; $\mathrm{TCX}=$ temporal cortex; $\mathrm{IHC}=$ immunohistochemistry.

$4^{\circ} \mathrm{C}$. Finally, sections were rinsed and incubated for $1 \mathrm{~h}$ with Alexa Fluor-conjugated secondary antibodies (Invitrogen) at room temperature. Images were collected with a $63 \times$ oil-immersion objective on a Zeiss LSM510 confocal microscope.

\section{Statistical analysis}

Data collected in this study were tested for normality using the Kolmogorov-Smirnov test (Prism 7, GraphPad). Estimation-based confidence intervals (Cls) were calculated using the DABEST estimation statistics package with the permutation $t$ test in Python 3.7.6 (Ho et al., 2019). For each estimation plot, (1) raw data were plotted on the upper axes (or left side) with their mean \pm SD indicated by the vertical gapped lines; and (2) their effect size was plotted on the lower or right floating axes with the mean difference and $95 \% \mathrm{Cls}$ indicated by dot and the ends of the vertical error bar, respectively. For each permutation $t$ test, 5000 bootstrap samples were taken. The $\mathrm{Cl}$ was bias-corrected and accelerated, and the resulting $p$ value is the likelihood of observing the effect size, if the null hypothesis of zero difference is true. The statistical tests applied in each figure are summarized in Table 3. We also performed unpaired (two-tailed) Student's $t$ tests for the RNA-seq data analysis (Table 4). A $\chi^{2}$ test was used to compare the gender and APOE4 composition between the AD and control groups (Tables 1, 2).

\section{Results}

To investigate the relative expression of $\mathrm{m} 6 \mathrm{~A}$ regulators in the postmortem AD brain, we first analyzed a publicly available transcriptomic dataset from the Allen Institute's Aging, Dementia and TBI Study (Miller et al., 2017). A total of 23 genes with known functions in the m6A signaling pathway, including $\mathrm{m} 6 \mathrm{~A}$ writers, erasers, and readers (Huang et al., 2020b), were examined based on their levels of expression in three distinct brain regions (hippocampus, parietal cortex, and temporal cortex) of $A D$ patients relative to healthy age-matched and sexmatched subjects (Table 1). Using unpaired (two-tailed)
Student's $t$ tests, we identified a number of genes which were differentially expressed in the $A D$ subjects relative to the control group as summarized in Table 4. Significantly dysregulated genes included METTL3 (downregulated in the hippocampus), $R B M 15 B$ (upregulated in all brain regions), $A L K B H 5$ and ELVAL3 (upregulated in the temporal cortex), and HNRNPA2B1 (upregulated in the parietal cortex). Interestingly, METTL3 (Fig. 1A) and RBM15B (Fig. $1 B)$, both of which are essential in m6A deposition, were the only two transcripts identified as being differentially expressed in the hippocampus, a key region of the brain for learning and memory.

To determine whether AD-associated changes in the m6A writer complex could be detected at the protein level, we obtained hippocampal tissues from an independent cohort of human postmortem $A D$ and control subjects (Table 2; Extended Data Table 2-1). Western blot analysis revealed a significant downregulation in the level of detergent-soluble METTL3 (Fig. 2A,B), while the level of RBM15B was markedly increased in the AD subjects compared with the control group (Fig. $2 A, C$ ). These data were consistent with the changes observed at the transcript level (Fig. 1). No significant changes were observed in the levels of METTL14 (Fig. 2A,D) and Tau proteins (Fig. $2 A, E)$ in the soluble fractions of the AD samples.

Proteinopathy in AD is often associated with the aggregation of proteins such as Tau, which accumulate in the insoluble fraction. To examine whether any of the m6A writers underwent aberrant aggregation in the hippocampal tissues of $A D$ subjects, we isolated the detergent-insoluble fractions derived from these samples. We did not detect the presence of RBM15B or METTL14 in the insoluble fractions of any of the control or AD samples (Fig. $3 A)$. Surprisingly, however, we observed a significant increase in the level of METTL3 in the insoluble fractions derived from the hippocampal tissues of $A D$ patients relative to the control group (Fig. $3 A, B$ ). As expected, the AD hippocampal tissues also contained a significantly greater accumulation of insoluble Tau protein compared with the control group (Fig. 3A,C). Interestingly, the level of METTL3 positively correlated with that of Tau in the 
Table 4: Summary data of m6A-related gene profiles in control versus AD subjects

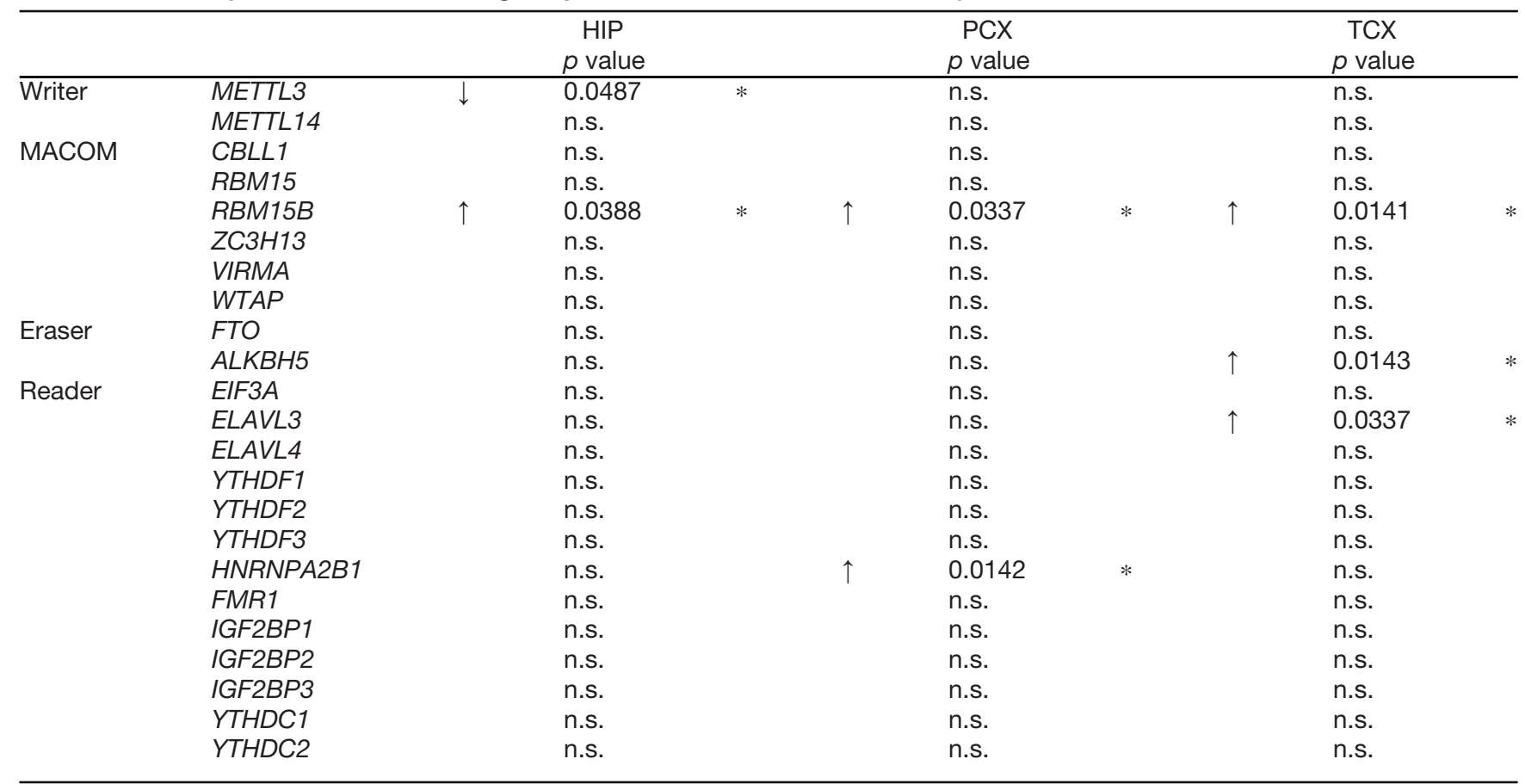

HIP = hippocampus; PCX = parietal cortex; TCX = temporal cortex; MACOM = m6A-methyltransferase associated complex.

$\uparrow$ and $\downarrow$ indicate an increase and decrease, respectively, in the gene expression pattern in AD compared with control subjects.

n.s. = not significant; $* p<0.05$; unpaired $t$ test.

insoluble fraction of these hippocampal lysates (Fig. $3 D$ ). However, the same analysis revealed no correlation between the level of insoluble METTL3 with the levels of $A \beta 40$ or $A \beta 42$ in the hippocampus (Extended Data Fig. 3-1).

To examine the distribution of METTL3, we performed immunohistochemical analyses on the paraformaldehyde-fixed hippocampal sections obtained from $A D$ and control subjects (Fig. $4 A, B$ ). The lack of staining in an $A D$ hippocampal section performed in the absence of anti-
METTL3 confirmed the specificity of the immunohistochemistry procedure (Fig. 4C). In control tissues, relatively higher expression of METTL3 was apparent in the CA2 region (Fig. 4A). However, we observed more pronounced staining throughout the hippocampal formation, particularly in the DG, in the AD tissues (Fig. 4B). Quantitative OD measurement of the DAB staining confirmed significantly higher METTL3 immunoreactivity in all subregions of the hippocampal tissues of $A D$ subjects relative to the controls (Fig. 4D).
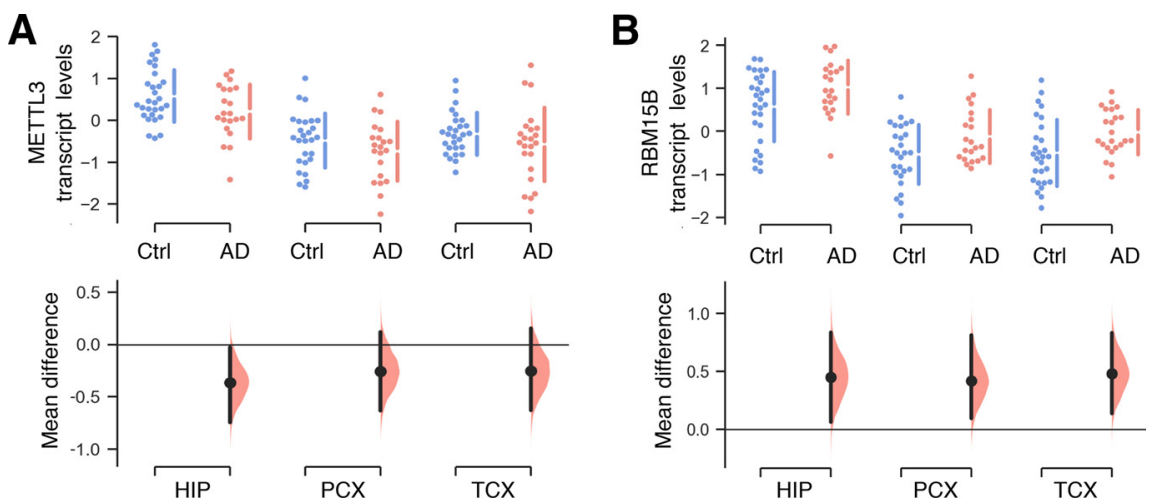

Figure 1. Altered expression of METTL3 and RBM15B transcripts in the AD brain. In silico analyses of METTL3 (A) and RBM15B (B) mRNA expression in various postmortem human brain tissues from AD patients (pink dots) and control subjects (blue dots) from the Allen Institute for Brain Science transcriptomic database. Raw data are shown on the upper axes. Estimation plots below display the mean differences between the AD and control groups. METTL3 $\left(\mathrm{HIP}, \mathrm{M}_{\text {diff }}=-0.37[95 \% \mathrm{Cl}-0.75,-0.03], p=0.049 ; \mathrm{PCX}, \mathrm{M}_{\text {diff }}=\right.$ $\left.-0.26[95 \% \mathrm{Cl}-0.63,0.12], p=0.204 ; \mathrm{TCX}, \mathrm{M}_{\text {diff }}=-0.25[95 \% \mathrm{Cl}-0.63,0.15], p=0.220\right) . \mathrm{RBM} 15 \mathrm{~B}(\mathrm{HIP}, \mathrm{M}$ diff $=0.45[95 \% \mathrm{Cl}$ $0.06,0.83$ ],$p=0.040 ; \mathrm{PCX}, \mathrm{M}_{\text {diff }}=0.42$ [95\% $\left.\left.0.09,0.81\right], p=0.032 ; \mathrm{TCX}, \mathrm{M}_{\text {diff }}=0.48[95 \% \mathrm{Cl} 0.13,0.83], p=0.012\right)$. HIP, hippocampus (control, $n=29$; AD, $n=21$ ); PCX, parietal cortex (control, $n=27 ; \mathrm{AD}, n=21)$; TCX, temporal cortex (control, $n=28 ; \mathrm{AD}, n=23$ ). 
A

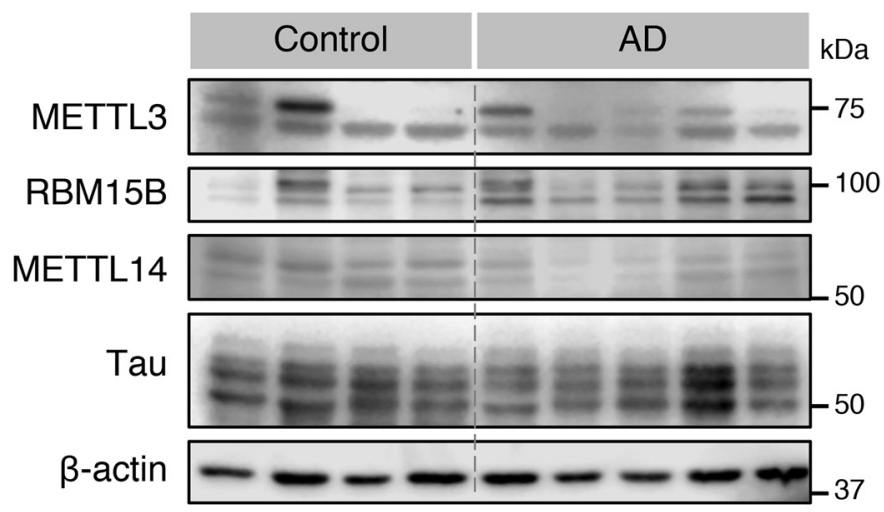

B

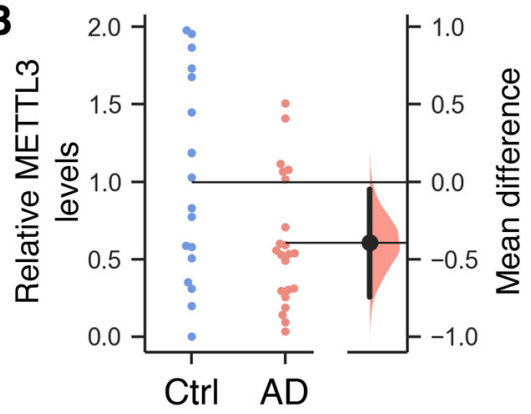

D

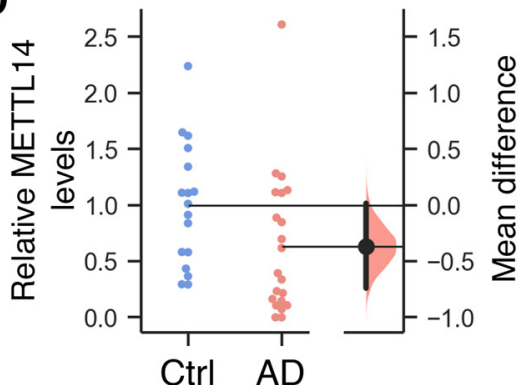

C

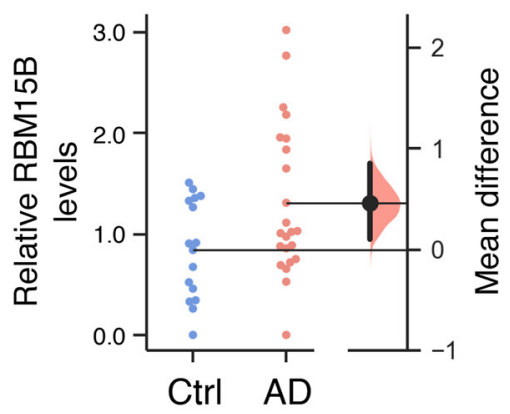

E

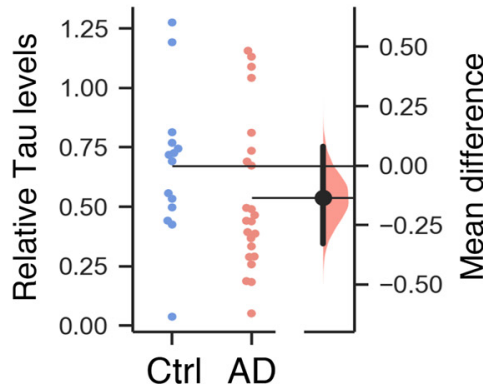

Figure 2. Analyses of METTL3, RBM15B, and METTL14 protein levels in the soluble fractions of postmortem AD hippocampal tissues. $\boldsymbol{A}$, Postmortem hippocampal tissue from AD patients and control subjects from the Alzheimer's Disease Research Center of the University of California, Irvine, was lysed in T-PER buffer. Detergent-soluble fractions of the lysates were subjected to SDS-PAGE and Western blotting with specific antibodies against METTL3, RBM15B, METTL14, Tau, and $\beta$-actin. Representative blots are shown. Densitometry analyses of the blots for METTL3 $(\boldsymbol{B})$, RBM15B $(\boldsymbol{C})$, METTL14 $(\boldsymbol{D})$, and Tau $(\boldsymbol{E})$ after normalization with $\beta$-actin are presented as estimation plots. Raw data are plotted on the left (control, blue dots; AD, pink dots), and mean differences are shown on the right. METTL3 $\left(\mathrm{M}_{\text {diff }}=-0.39[95 \% \mathrm{Cl}-0.75,-0.05], p=0.030\right)$, RBM15B $\left(\mathrm{M}_{\text {diff }}=0.46[95 \% \mathrm{Cl} 0.11,0.85], p=0.036\right), \mathrm{METTL14}(\mathrm{M}$ diff $=$ $-0.37[95 \% \mathrm{Cl}-0.74,0.02], p=0.074)$ and Tau ( $\left.\mathrm{M}_{\text {diff }}=-0.13[95 \% \mathrm{Cl}-0.33,0.08], p=0.219\right)$. AD $(n=21-23)$, control $(n=12-17)$.

The positive correlation in the levels of METTL3 and Tau in the insoluble fractions (Fig. 3) also prompted us to investigate the potential interaction between these two proteins. To address this, we co-transfected human Tau-V5, either wild-type or the AD-associated P301L mutant, with myc-Mettl3 plasmids in HEK293T cells and performed co-immunoprecipitation assays. As expected, endogenous METTL14 co-immunoprecipitated with myc-Mett|3, validating the specificity of the assay (Fig. 4E). However, co-immunoprecipitation using specific antibodies against myc revealed no interactions between Tau-V5 and myc-Mett|3 (Fig. 4E). Furthermore, immunofluorescence staining of METTL3 and Tau revealed a distinct subcellular localization of these two proteins in $A D$ hippocampal tissues (Fig. 4F). Tau appeared as fibrillar structures, which is one of the pathologic hallmarks of $A D$. On the other hand, METTL3 was detected in the cell bodies of MAP2-positive neurons that did not possess intracellular neurofibrillary tangles. Collectively, these data suggest that although Tau pathology is a better predictor of deregulated m6A signaling than $A \beta$ load, it is unlikely to play a causal role in altering METTL3 expression in the hippocampus of $A D$ patients.

\section{Discussion}

The m6A epitranscriptome adds to the complex regulation of RNA metabolism and function in neurons, 
A

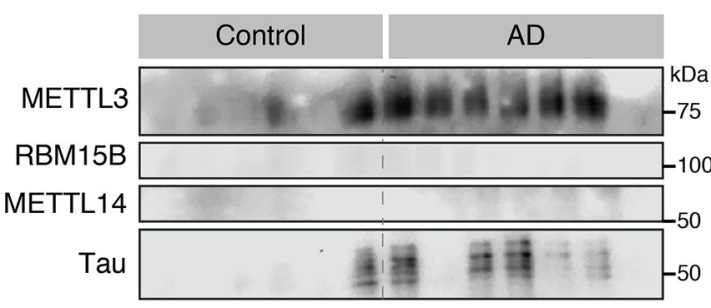

B

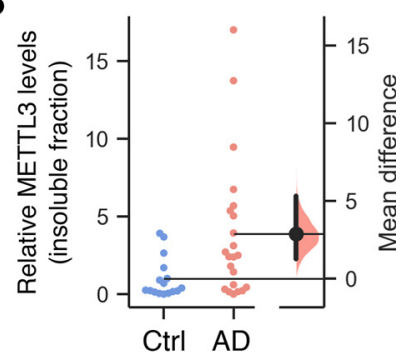

C

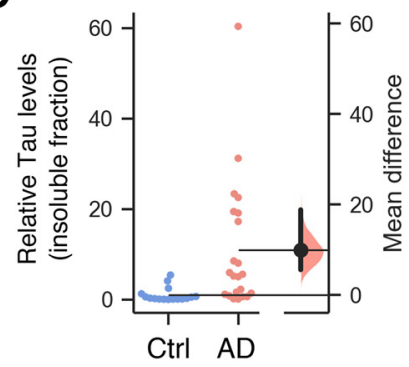

D

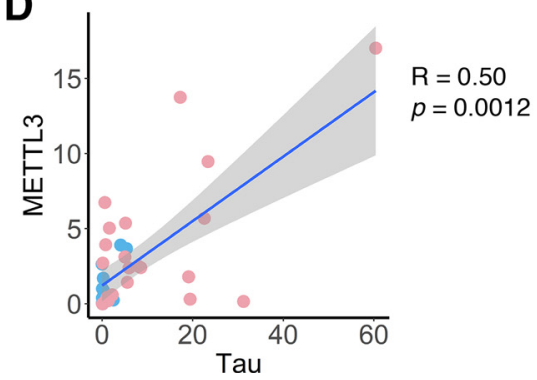

Figure 3. Elevated level of METTL3 protein in the insoluble fraction of human postmortem AD hippocampal tissues. A, Representative Western blots of detergent-insoluble fractions prepared from postmortem hippocampal tissue from AD patients and control subjects, probed with specific antibodies against METTL3, RBM15B, METTL14, and Tau. Densitometry analyses of the blots for METTL3 $(\boldsymbol{B})$ and Tau $(\boldsymbol{C})$ are presented as estimation plots. Raw data are plotted on the left (control, blue dots; AD, pink dots), and mean differences are shown on the right. METTL3 $\left(\mathrm{M}_{\text {diff }}=2.87[95 \% \mathrm{Cl} 1.27,5.31], p=0.012\right)$ and Tau $\left(\mathrm{M}_{\text {diff }}=9.92[95 \% \mathrm{Cl}\right.$ $5.53,18.76], p=0.003)$. AD $(n=22)$, control $(n=16)$. $\boldsymbol{D}$. Accumulation of METTL3 in the insoluble fraction of human hippocampal tissues positively correlates with Tau aggregates (control, blue dots; AD, pink dots; Spearman's correlation coefficient $=0.50$, $p=0.0012)$.

rendering its essential roles in neuronal development, synaptic plasticity, cognition and the stress response (Engel and Chen, 2018; Huang and Lu, 2018; Widagdo and Anggono, 2018; Flamand and Meyer, 2019). The m6A methylome is dynamically regulated by sensory and learning experience in the hippocampus, striatum, and cortex of mice (Widagdo et al., 2016; Engel et al., 2018; Koranda et al., 2018; Zhang et al., 2018). Activity-dependent m6A deposition occurs on many transcripts of synaptic plasticity-related and immediate early genes (Widagdo et al., 2016; Shi et al., 2018; Zhang et al., 2018). Mice lacking the m6A writers Mettl3 or Mettl14 exhibit impairments in synaptic plasticity, learning, and memory consolidation (Koranda et al., 2018; Zhang et al., 2018). In contrast, increasing the abundance of $\mathrm{m} 6 \mathrm{~A}$ by knocking down the expression of the m6A demethylating enzyme, FTO, promotes memory consolidation in mice (Widagdo et al., 2016; Walters et al., 2017).

Alterations in RNA modifying enzymes have been widely implicated in human diseases, underscoring the importance of post-transcriptional control of RNA function in general physiology (Kadumuri and Janga, 2018). Dysregulation of global m6A abundance and aberrant expression of $\mathrm{m} 6 \mathrm{~A}$ writers, erasers, and readers are strongly associated with the pathophysiology of various cancer types and play critical roles in their initiation, progression, metastasis, and relapse ( $\mathrm{Li}$ et al., 2019; Huang et al., 2020a). However, there has been little investigation of the epitranscriptomic changes that are associated with human neurodegenerative disorders. In genome-wide association studies, a gene variant in the $\mathrm{m} 6 \mathrm{~A}$ demethylase
FTO has been reported to be a risk factor in AD (Ho et al., 2010; Keller et al., 2011), but the functional implication of this remains unclear. Here, we provide the first demonstration of dysregulated expression of the key m6A methyltransferase METTL3 in the human postmortem AD brain, indicating an impairment in $\mathrm{m} 6 \mathrm{~A}$ signaling in the pathophysiology of AD.

In mice, loss of METTL3 m6A methyltransferase function in the hippocampus has a negative impact on memory consolidation (Zhang et al., 2018). Downregulation of METTL3 mRNA and soluble protein levels in the AD hippocampus may therefore correlate with the memory dysfunction associated with this disease. The suppression of METTL3 function is further indicated by its increased insolubility, which positively correlates with the presence of Tau aggregates, but not with $\mathrm{A} \beta$ load or ApoE4 genotype (data not shown). This, accompanied by an elevated level and redistribution of METTL3 expression in AD hippocampal tissue, likely represents abnormal misfolding and/or aggregation of METTL3, perhaps resembling the frequent aggregation of RNA-binding proteins in neurodegenerative disorders (Arai et al., 2009; Conlon and Manley, 2017). Given that several $m 6 A$ regulatory proteins are subjected to post-translational ubiquitination and SUMOylation (Zhang et al., 2015; Tai et al., 2017; Du et al., 2018; Zhu et al., 2018; Liu et al., 2020; Xu et al., 2020), it is conceivable that dysregulation of the ubiquitin-proteasome system may contribute to the aggregation of METTL3 in AD (Ihara et al., 2012; Zheng et al., 2016).

Interestingly, our findings are in stark contrast with a recent study that reported an increase in the mRNA and 
A

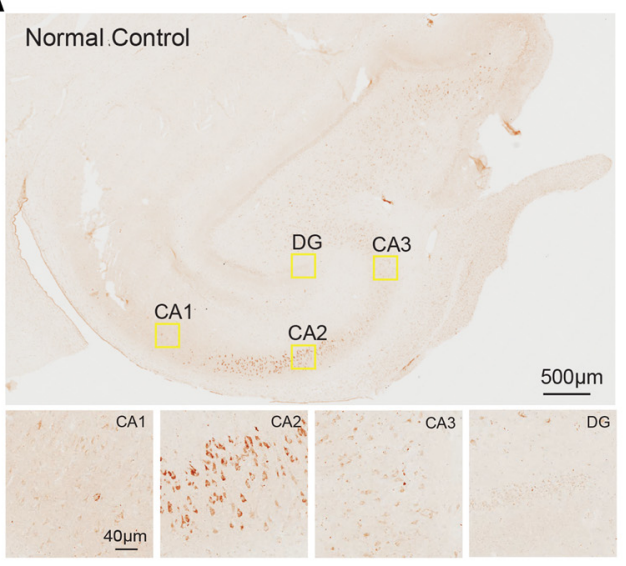

B

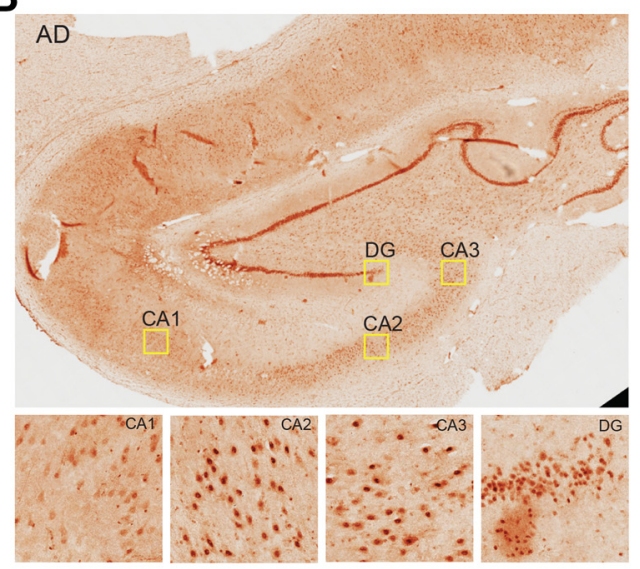

C

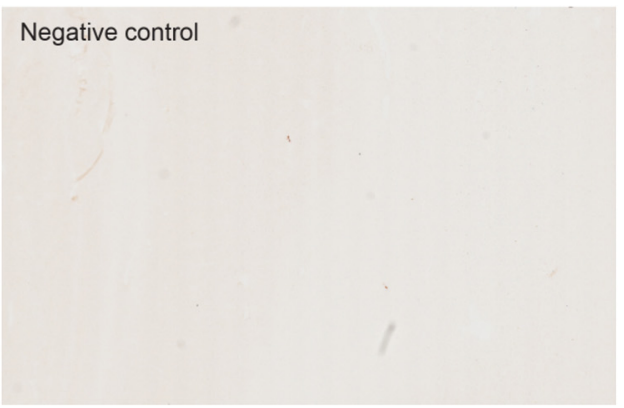

D
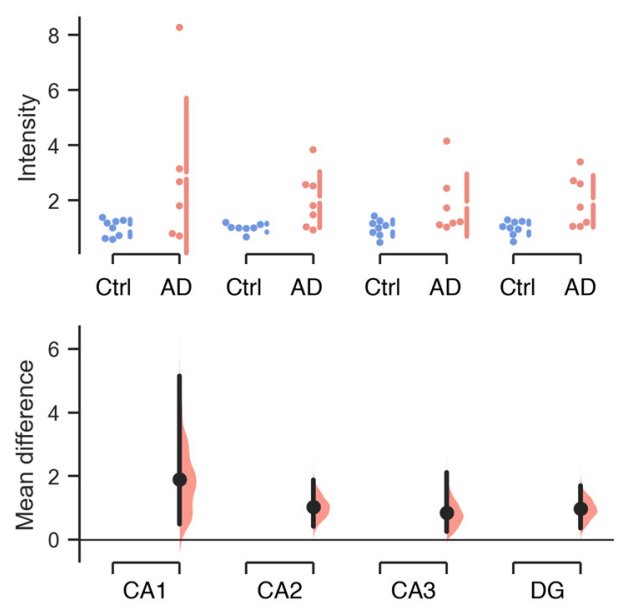

E

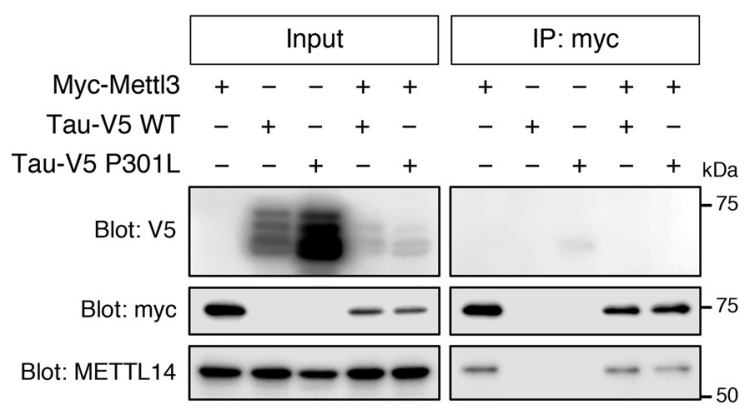

$\mathbf{F}$

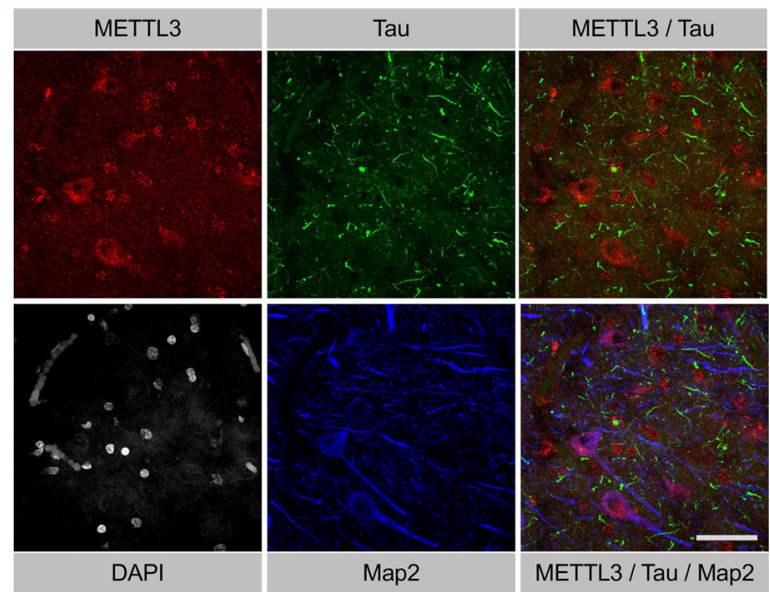

Figure 4. Analysis of METTL3 distribution and localization in postmortem human AD hippocampal tissue. Representative immunohistochemical staining of METTL3 in the human postmortem AD $(\boldsymbol{A})$ and control hippocampal tissues (B). Magnified images of the selected regions are shown below. CA, cornu ammonis. Scale bar $=500 \mu \mathrm{m}$ (low-power images) or $40 \mu \mathrm{m}$ (high-power images). $\boldsymbol{C}$, Negative staining performed on AD hippocampal tissue using the same protocol without the primary antibody against METTL3. $\boldsymbol{D}$, Quantification of METTL3 OD measurement and mean differences in $\mathrm{CA} 1\left(\mathrm{M}_{\text {diff }}=1.90[95 \% \mathrm{Cl} 0.48,5.15], p=0.015\right), \mathrm{CA} 2(\mathrm{M}$ diff $=$ $1.03[95 \% \mathrm{Cl} 0.42,1.87], p=0.014)$, CA3 $\left(\mathrm{M}_{\text {diff }}=0.84[95 \% \mathrm{Cl} 0.25,2.11], p=0.025\right)$ and the $\mathrm{DG}\left(\mathrm{M}_{\text {diff }}=0.97[95 \% \mathrm{Cl} 0.36,1.70]\right.$, $p=0.007)$. Data are presented as estimation plots, AD $(n=6-7)$, control $(n=7-8)$. $\boldsymbol{E}$, Mettl3 does not interact with Tau in cells. HEK293T cells were transfected with the indicated plasmids for $48 \mathrm{~h}$, lysed and immunoprecipitated with anti-myc antibodies. Bound proteins were eluted and resolved by SDS-PAGE, and analyzed by Western blottings with specific antibodies against myc, V5 and METTL14. F, Immunofluorescence staining revealed that METTL3 (red) and Tau (green) do not colocalize in AD hippocampal tissue. MAP2 and DAPI were used to stain for neuronal dendrites (blue) and nuclei (gray), respectively. Scale bar $=50 \mu \mathrm{m}$ 
protein expression of Mettl3 in the APP/PS1 mouse model of AD (Han et al., 2020). A concomitant decrease in the levels of FTO transcript and protein was also observed, resulting in a net increase in $\mathrm{m} 6 \mathrm{~A}$ abundance in the cortex and hippocampus of these mice. This discrepancy could simply be because of the use of different model systems (postmortem human $A D$ brains vs an $A D$ mouse model). However, given that we did not see a correlation between the alteration of METTL3 expression with $\mathrm{A} \beta$ load, future studies using Tau mouse models are warranted.

METTL3 and METTL14 form a stable dimer in vivo, and perturbation in one component leads to instability of the m6A-writer complex (Liu et al., 2014). Our analysis did not find alteration in the mRNA level of METTL14 in AD despite a trend toward a reduced METTL14 protein level in the soluble fraction. However, the increase in the mRNA and protein levels of RBM15B, a key member of the MACOM that recruits METTL3/14 to mRNA (Patil et al., 2016), may reflect a compensatory response to the downregulation of the functional core $\mathrm{m} 6 \mathrm{~A}$-methyltransferase complex in the brain. Overall, our study demonstrates dysregulation of key $\mathrm{m} 6 \mathrm{~A}$ regulatory enzymes in the human postmortem AD brain, implicating a perturbation in $\mathrm{m} 6 \mathrm{~A}$ signaling in the pathology of the disease, a possibility that warrants further investigation.

\section{References}

Anggono V, Koç-Schmitz Y, Widagdo J, Kormann J, Quan A, Chen CM, Robinson PJ, Choi SY, Linden DJ, Plomann M, Huganir RL (2013) PICK1 interacts with PACSIN to regulate AMPA receptor internalization and cerebellar long-term depression. Proc Natl Acad Sci USA 110:13976-13981.

Arai T, Mackenzie IR, Hasegawa M, Nonoka T, Niizato K, Tsuchiya K, Iritani S, Onaya M, Akiyama H (2009) Phosphorylated TDP-43 in Alzheimer's disease and dementia with Lewy bodies. Acta Neuropathol 117:125-136.

Conlon EG, Manley JL (2017) RNA-binding proteins in neurodegeneration: mechanisms in aggregate. Genes Dev 31:1509-1528.

De Jager PL, Srivastava G, Lunnon K, Burgess J, Schalkwyk LC, Yu L, Eaton ML, Keenan BT, Ernst J, McCabe C, Tang A, Raj T, Replogle J, Brodeur W, Gabriel S, Chai HS, Younkin C, Younkin SG, Zou F, Szyf M, et al. (2014) Alzheimer's disease: early alterations in brain DNA methylation at ANK1, BIN1, RHBDF2 and other loci. Nat Neurosci 17:1156-1163.

Du Y, Hou G, Zhang H, Dou J, He J, Guo Y, Li L, Chen R, Wang Y, Deng R, Huang J, Jiang B, Xu M, Cheng J, Chen GQ, Zhao X, Yu J (2018) SUMOylation of the m6A-RNA methyltransferase METTL3 modulates its function. Nucleic Acids Res 46:5195-5208.

Engel M, Chen A (2018) The emerging role of mRNA methylation in normal and pathological behavior. Genes Brain Behav 17:e12428.

Engel M, Eggert C, Kaplick PM, Eder M, Röh S, Tietze L, Namendorf C, Arloth J, Weber P, Rex-Haffner M, Geula S, Jakovcevski M, Hanna JH, Leshkowitz D, Uhr M, Wotjak CT, Schmidt MV, Deussing JM, Binder EB, Chen A (2018) The role of $\mathrm{m}(6) \mathrm{A} / \mathrm{m}-\mathrm{RNA}$ methylation in stress response regulation. Neuron 99:389-403.

Flamand MN, Meyer KD (2019) The epitranscriptome and synaptic plasticity. Curr Opin Neurobiol 59:41-48.

Han M, Liu Z, Xu Y, Liu X, Wang D, Li F, Wang Y, Bi J (2020) Abnormality of m6A mRNA methylation is involved in Alzheimer's disease. Front Neurosci 14:98.

Ho AJ, Stein JL, Hua X, Lee S, Hibar DP, Leow AD, Dinov ID, Toga AW, Saykin AJ, Shen L, Foroud T, Pankratz N, Huentelman MJ, Craig DW, Gerber JD, Allen AN, Corneveaux JJ, Stephan DA,
DeCarli CS, DeChairo BM, et al. (2010) A commonly carried allele of the obesity-related FTO gene is associated with reduced brain volume in the healthy elderly. Proc Natl Acad Sci USA 107:84048409.

Ho J, Tumkaya T, Aryal S, Choi H, Claridge-Chang A (2019) Moving beyond $P$ values: data analysis with estimation graphics. Nat Methods 16:565-566.

Huang $\mathrm{H}$, Weng $\mathrm{H}$, Chen $\mathrm{J}$ (2020a) m(6)A modification in coding and non-coding RNAs: roles and therapeutic implications in cancer. Cancer Cell 37:270-288.

Huang $\mathrm{H}$, Weng $\mathrm{H}$, Chen $\mathrm{J}(2020 \mathrm{~b})$ The biogenesis and precise control of RNA m(6)A methylation. Trends Genet 36:44-52.

Huang YS, Lu WH (2018) Decoding hidden messages in neurons: insights from epitranscriptome-controlled and specialized ribosome-controlled translation. Curr Opin Neurobiol 48:64-70.

Ihara Y, Morishima-Kawashima M, Nixon R (2012) The ubiquitin-proteasome system and the autophagic-lysosomal system in Alzheimer disease. Cold Spring Harb Perspect Med 2:a006361.

Jia G, Fu Y, Zhao X, Dai Q, Zheng G, Yang Y, Yi C, Lindahl T, Pan T, Yang YG, He C (2011) N6-methyladenosine in nuclear RNA is a major substrate of the obesity-associated FTO. Nat Chem Biol 7:885-887.

Kadumuri RV, Janga SC (2018) Epitranscriptomic code and its alterations in human disease. Trends Mol Med 24:886-903.

Keller L, Xu W, Wang HX, Winblad B, Fratiglioni L, Graff C (2011) The obesity related gene, FTO, interacts with APOE, and is associated with Alzheimer's disease risk: a prospective cohort study. J Alzheimers Dis 23:461-469.

Klein HU, McCabe C, Gjoneska E, Sullivan SE, Kaskow BJ, Tang A, Smith RV, Xu J, Pfenning AR, Bernstein BE, Meissner A, Schneider JA, Mostafavi S, Tsai LH, Young-Pearse TL, Bennett DA, De Jager PL (2019) Epigenome-wide study uncovers large-scale changes in histone acetylation driven by tau pathology in aging and Alzheimer's human brains. Nat Neurosci 22:37-46.

Koranda JL, Dore L, Shi H, Patel MJ, Vaasjo LO, Rao MN, Chen K, Lu Z, Yi Y, Chi W, He C, Zhuang X (2018) Mettl14 is essential for epitranscriptomic regulation of striatal function and learning. Neuron 99:283-292.

Li C, Götz J (2017) Somatodendritic accumulation of Tau in Alzheimer's disease is promoted by Fyn-mediated local protein translation. EMBO J 36:3120-3138.

Li L, Zang L, Zhang F, Chen J, Shen H, Shu L, Liang F, Feng C, Chen D, Tao H, Xu T, Li Z, Kang Y, Wu H, Tang L, Zhang P, Jin P, Shu Q, Li X (2017) Fat mass and obesity-associated (FTO) protein regulates adult neurogenesis. Hum Mol Genet 26:2398-2411.

Li Y, Xiao J, Bai J, Tian Y, Qu Y, Chen X, Wang Q, Li X, Zhang Y, Xu J (2019) Molecular characterization and clinical relevance of $m(6) A$ regulators across 33 cancer types. Mol Cancer 18:137.

Liu J, Yue Y, Han D, Wang X, Fu Y, Zhang L, Jia G, Yu M, Lu Z, Deng $X$, Dai Q, Chen W, He C (2014) A METTL3-METTL14 complex mediates mammalian nuclear RNA N6-adenosine methylation. Nat Chem Biol 10:93-95.

Liu X, Liu J, Xiao W, Zeng Q, Bo H, Zhu Y, Gong L, He D, Xing X, Li R, Zhou M, Xiong W, Zhou Y, Zhou J, Li X, Guo F, Xu C, Chen X, Wang X, Wang F, Wang Q, Cao K (2020) SIRT1 regulates N(6) -methyladenosine RNA modification in hepatocarcinogenesis by inducing RANBP2-dependent FTO SUMOylation. Hepatology, in press.

Livneh I, Moshitch-Moshkovitz S, Amariglio N, Rechavi G, Dominissini D (2020) The m(6)A epitranscriptome: transcriptome plasticity in brain development and function. Nat Rev Neurosci 21:36-51.

Lunnon K, Smith R, Hannon E, De Jager PL, Srivastava G, Volta M, Troakes C, Al-Sarraj S, Burrage J, Macdonald R, Condliffe D, Harries LW, Katsel P, Haroutunian V, Kaminsky Z, Joachim C, Powell J, Lovestone S, Bennett DA, Schalkwyk LC, et al. (2014) Methylomic profiling implicates cortical deregulation of ANK1 in Alzheimer's disease. Nat Neurosci 17:1164-1170.

Marzi SJ, Leung SK, Ribarska T, Hannon E, Smith AR, Pishva E, Poschmann J, Moore K, Troakes C, Al-Sarraj S, Beck S, Newman 
S, Lunnon K, Schalkwyk LC, Mill J (2018) A histone acetylomewide association study of Alzheimer's disease identifies diseaseassociated H3K27ac differences in the entorhinal cortex. Nat Neurosci 21:1618-1627.

Medeiros R, Castello NA, Cheng D, Kitazawa M, Baglietto-Vargas D, Green KN, Esbenshade TA, Bitner RS, Decker MW, LaFerla FM (2014) $\alpha 7$ nicotinic receptor agonist enhances cognition in aged 3xTg-AD mice with robust plaques and tangles. Am J Pathol 184:520-529.

Miller JA, Guillozet-Bongaarts A, Gibbons LE, Postupna N, Renz A, Beller AE, Sunkin SM, Ng L, Rose SE, Smith KA, Szafer A, Barber C, Bertagnolli D, Bickley K, Brouner K, Caldejon S, Chapin M, Chua ML, Coleman NM, Cudaback E, et al. (2017) Neuropathological and transcriptomic characteristics of the aged brain. Elife 6:e31126.

Nativio R, Donahue G, Berson A, Lan Y, Amlie-Wolf A, Tuzer F, Toledo JB, Gosai SJ, Gregory BD, Torres C, Trojanowski JQ, Wang LS, Johnson FB, Bonini NM, Berger SL (2018) Dysregulation of the epigenetic landscape of normal aging in Alzheimer's disease. Nat Neurosci 21:497-505.

Neueder A (2019) RNA-mediated disease mechanisms in neurodegenerative disorders. J Mol Biol 431:1780-1791.

Patil DP, Chen C-K, Pickering BF, Chow A, Jackson C, Guttman M, Jaffrey SR (2016) m(6)A RNA methylation promotes XIST-mediated transcriptional repression. Nature 537:369-373.

Ping XL, Sun BF, Wang L, Xiao W, Yang X, Wang WJ, Adhikari S, Shi Y, Lv Y, Chen YS, Zhao X, Li A, Yang Y, Dahal U, Lou XM, Liu X, Huang J, Yuan WP, Zhu XF, Cheng T, et al. (2014) Mammalian WTAP is a regulatory subunit of the RNA N6-methyladenosine methyltransferase. Cell Res 24:177-189.

Polanco JC, Li C, Bodea L-G, Martinez-Marmol R, Meunier FA, Götz $\mathrm{J}$ (2018) Amyloid- $\beta$ and tau complexity - towards improved biomarkers and targeted therapies. Nat Rev Neurol 14:22-39.

Saletore Y, Meyer K, Korlach J, Vilfan ID, Jaffrey S, Mason CE (2012) The birth of the epitranscriptome: deciphering the function of RNA modifications. Genome Biol 13:175.

Sanchez-Mut JV, Graff J (2015) Epigenetic alterations in Alzheimer's disease. Front Behav Neurosci 9:347.

Schwartz S (2016) Cracking the epitranscriptome. RNA 22:169-174.

Shi H, Zhang X, Weng YL, Lu Z, Liu Y, Lu Z, Li J, Hao P, Zhang Y, Zhang F, Wu Y, Delgado JY, Su Y, Patel MJ, Cao X, Shen B, Huang X, Ming GL, Zhuang X, Song H, et al. (2018) m(6)A facilitates hippocampus-dependent learning and memory through YTHDF1. Nature 563:249-253.

Shi H, Wei J, He C (2019) Where, when, and how: context-dependent functions of RNA methylation writers, readers, and erasers. Mol Cell 74:640-650.

Tai H, Wang X, Zhou J, Han X, Fang T, Gong H, Huang N, Chen H, Qin J, Yang M, Wei X, Yang L, Xiao H (2017) Protein kinase $\mathrm{C} \beta$ activates fat mass and obesity-associated protein by influencing its ubiquitin/proteasome degradation. FASEB J 31:4396-4406.
Tarawneh R, Holtzman DM (2012) The clinical problem of symptomatic Alzheimer disease and mild cognitive impairment. Cold Spring Harb Perspect Med 2:a006148.

Walters BJ, Mercaldo V, Gillon CJ, Yip M, Neve RL, Boyce FM, Frankland PW, Josselyn SA (2017) The role of the RNA demethylase FTO (fat mass and obesity-associated) and mRNA methylation in hippocampal memory formation. Neuropsychopharmacology 42:1502-1510.

Widagdo J, Anggono V (2018) The m6A-epitranscriptomic signature in neurobiology:from neurodevelopment to brain plasticity. J Neurochem 147:137-152.

Widagdo J, Zhao QY, Kempen MJ, Tan MC, Ratnu VS, Wei W, Leighton L, Spadaro PA, Edson J, Anggono V, Bredy TW (2016) Experience-dependent accumulation of N6-methyladenosine in the prefrontal cortex is associated with memory processes in mice. J Neurosci 36:6771-6777.

Xu H, Wang H, Zhao W, Fu S, Li Y, Ni W, Xin Y, Li W, Yang C, Bai Y, Zhan M, Lu L (2020) SUMO1 modification of methyltransferaselike 3 promotes tumor progression via regulating Snail mRNA homeostasis in hepatocellular carcinoma. Theranostics 10:56715686.

Yang Y, Hsu PJ, Chen YS, Yang YG (2018) Dynamic transcriptomic m(6)A decoration: writers, erasers, readers and functions in RNA metabolism. Cell Res 28:616-624.

Zhang L, Tran NT, Su H, Wang R, Lu Y, Tang H, Aoyagi S, Guo A, Khodadadi-Jamayran A, Zhou D, Qian K, Hricik T, Côté J, Han X, Zhou W, Laha S, Abdel-Wahab O, Levine RL, Raffel G, Liu Y, et al. (2015) Cross-talk between PRMT1-mediated methylation and ubiquitylation on RBM15 controls RNA splicing. Elife 4:e07938.

Zhang Z, Wang M, Xie D, Huang Z, Zhang L, Yang Y, Ma D, Li W, Zhou Q, Yang YG, Wang XJ (2018) METTL3-mediated N(6)-methyladenosine mRNA modification enhances long-term memory consolidation. Cell Res 28:1050-1061.

Zhao BS, Roundtree IA, He C (2017) Post-transcriptional gene regulation by mRNA modifications. Nat Rev Mol Cell Biol 18:31-42.

Zheng G, Dahl JA, Niu Y, Fedorcsak P, Huang CM, Li CJ, Vågbø CB, Shi Y, Wang WL, Song SH, Lu Z, Bosmans RPG, Dai Q, Hao YJ, Yang X, Zhao WM, Tong WM, Wang XJ, Bogdan F, Furu K, et al. (2013) ALKBH5 is a mammalian RNA demethylase that impacts RNA metabolism and mouse fertility. Mol Cell 49:18-29.

Zheng Q, Huang T, Zhang L, Zhou Y, Luo H, Xu H, Wang X (2016) Dysregulation of ubiquitin-proteasome system in neurodegenerative diseases. Front Aging Neurosci 8:303.

Zhu T, Yong XLH, Xia D, Widagdo J, Anggono V (2018) Ubiquitination regulates the proteasomal degradation and nuclear translocation of the fat mass and obesity-associated (FTO) protein. J Mol Biol 430:363-371.

Zumkehr J, Rodriguez-Ortiz CJ, Cheng D, Kieu Z, Wai T, Hawkins C, Kilian J, Lim SL, Medeiros R, Kitazawa M (2015) Ceftriaxone ameliorates tau pathology and cognitive decline via restoration of glial glutamate transporter in a mouse model of Alzheimer's disease. Neurobiol Aging 36:2260-2271. 